is tiny) we naturally worry about the implications of free movement. It seems strange to fear the Greeks when they come bearing the gifts of EEC membership, since we have for so long depended on the medical fruits of the Indian subcontinent to nurture the NHS. Yet times have changed. Although the General Medical Council is now exercising much control and discrimination over which overseas medical qualifications it will recognise it has been robbed of all such authority in respect of the EEC. There is no inspection of continental medical schools, nor qualitative assessment; 5500 hours of instruction over six years, with EEC nationality, carries automatic entitlement to full registration. We know very little about the standard of the "average" medical graduate of Italy or West Germany; what we do know-the performance in the past in the language examination of some doctors from countries now exempted by their new EEC membership-is not too reassuring. And there is not, as yet, much information about the thoroughness with which health authorities are testing linguistic and professional competence before employing EEC doctors. We are left with an uncomfortable sense of lost control with little gained in exchange.

A survey of the EEC countries shows how many wayssome tried, some theoretical-there are of trying to control medical manpower: limiting entry to the medical schools, cutting down undergraduate numbers after the first year, regulating postgraduate training opportunities by specialty and area, restricting the scope of practice by specialist registration, controlling the emigration of doctors, throttling the inflow of foreign doctors, and fixing the retirement age. In addition may be found different attitudes to part time training and different labour markets leading sometimes to partial employment of doctors who cannot find enough work to maintain their competence. Some end up in a state of "intellectual unemployment," practising fringe medicine or doing paramedical work to make a living.

Language difficulties are a barrier to free movement of doctors-and so they should be, unless adequately overcome. On the other hand, the Common Market arrangement has required the levelling up of training and educational requirements. This makes our membership, and that of Ireland, important to medicine in the rest of the EEC. But we set a curious example, for all our great traditions. Much of our postgraduate training is appallingly badly organised, inefficient, and unnecessarily long. It is beset by a complex network of diploma examinations which, inconsistent in their purpose and timing as between specialties and even between colleges in the same specialty, are no basis on which the rest of western Europe could model itself-even if it wished to emulate our apparent love of such tests. We may have to learn how to reconcile our criteria for specialist or consultant status with the claims of EEC doctors who have not passed postgraduate examinations or complied with the rigours of our higher professional training and who may be none the worse for it. If the flexibility inherent in our colleges' requirements is fairly challenged and tested in this way it could help us to clarify our thinking about the purpose of accreditation and the means of achieving it. What is to be avoided at all costs within the EEC is any kind of legislation concerning postgraduate training requirements which threatens to impose even greater rigidity than we have at present. The concept of a "common trunk" of early postgraduate training is hopeful, if it can be properly developed, as also is the current anxiety within the EEC committees about the inherent impracticability of seeking to insist on the complete training of a specialist.

These issues were discussed at a symposium in Stresa in
1982 assembled at the invitation of the Fondazione Smith Kline. In three intensive days the group reviewed medical demography; student selection; undergraduate, postgraduate, and continuing education; and medical audit. The well prepared opening papers and the whole of the discussion are now available. ${ }^{1}$ One of the particular qualities of the meeting was that it brought together medical practitioners with health economists and administrators in roughly equal numbers, each main topic being introduced by a doctor and either an economist or an administrator. Alan Maynard, who as a health economist worked alongside the chairman Sir John Walton, had characteristically pointed things to say about audit, which he identified as the most important issue in the long run. There is perceptible evidence of progress, at least in terms of receptivity to the concept of audit, and keen interest in initiatives on standard setting and quality control.

At Stresa postgraduate training and medical manpower hung together naturally, as they always must. And they hanged together as they always will-in default of effective collaboration between the interests concerned. With the career structure and the employment of doctors we are all in the tumbril and we look to the health economists, either for last minute absolution or to spell out the terms on which our lives may be spared. If we all try simultaneously to solve the same problems in different ways we can easily nullify each other's efforts. Hence the overriding need for study of medical manpower control mechanisms within the EEC to achieve concerted action. Yet, like many of its constituent nations, the EEC has bodies dealing with manpower that dare not interfere with education, and bodies dealing with training which particularly exclude manpower from their remit. There are partial mechanisms for discussing the problems-but no mechanisms at all for solving them. The Stresa meeting was an important three days, but who will take notice of what was said? Over 18 months have already passed and the published report, sadly, is as fresh as ever.

JAMEs PARKhouse

Director,

Medical Careers Research Group,

Churchill Hospital,

Oxford OX3 7LJ

1 Walton J, Binns TB, eds. Medical education and manpower in the EEC. London: Macmillan Press, in association with Fondazione Smith Kline, Milan, Italy, 1984.

\section{Metoclopramide and tardive dyskinesia in the elderly}

Metoclopramide may cause several extrapyramidal side effects. Acute dystonic reactions, such as akathisia and spasmodic deviation of the eyes and hands, are more common in children ${ }^{1}$ and tend to occur early in the course of treatment. ${ }^{2}$ In adults they usually coincide with high peak concentrations and disappear rapidly once the drug is stopped. ${ }^{3}$ Early claims that metoclopramide did not cause parkinsonian-like symptoms ${ }^{4}$ have now been refuted. ${ }^{256}$ Parkinsonism induced by metoclopramide is more common in the elderly, has a more rapid onset than Parkinson's disease, and is usually quickly reversed when the drug is stopped.

The suspicion that metoclopramide might be associated with the development of dyskinesia was first raised in 1978,? and further reports have followed. ${ }^{28} \mathrm{~A}$ recent article in the 
$B M \mathcal{F}$ reported 11 patients with tardive dyskinesia associated with metoclopramide reported to the Swedish Adverse Drug Reaction Advisory Committee during 1977-81. ${ }^{9}$ The patients were all women with a mean age of 76 . The average duration of treatment before the onset of symptoms was 14 months, and in only four patients was the dose above the recommended limit of $0.5 \mathrm{mg} / \mathrm{kg}$ body weight. The Swedish authorities put the incidence of tardive dyskinesia in patients aged 70 or more having long term treatment at greater than one in 1000 .

The tardive dyskinesias are a group of neurological disorders producing persistent, repetitive, choreiform movements of voluntary muscles, most frequently of the faciolingual-buccal muscle groups, where they may resemble sucking, chewing, or lip smacking. They tend to disappear during sleep and are made worse by emotional stress and voluntary movements of the limbs. ${ }^{10} 11$ The symptoms may be mild ${ }^{12}$ and more upsetting to observers than to patients, ${ }^{13}$ but they are often severe, distressing, and incapacitating. Though by definition tardive dyskinesia occurs after medication with neuroleptic drugs, it often presents after treatment has been stopped. This is true also in the case of tardive dyskinesia associated with metoclopramide. ${ }^{29}$ Why should this be?

In addition to having cholinergic properties metoclopramide is a specific antagonist of dopamine 2 receptors in the brain, which are particularly associated with dopamine mediated inhibition of the release of prolactin. ${ }^{14}$ (Drugs such as haloperidol are antagonistic at the dopamine 1 receptors.) The tardive dyskinesias are possibly caused by hypersensitivity of the dopamine receptors in the corpus striatum, so explaining why they may improve if the dose of metoclopramide is increased, since this will restore the blockade of dopamine 2 receptors. ${ }^{\text {is }}$ Increasing the dose is not, however, good clinical practice because a vicious circle will result. The hypersensitivity theory also explains why metoclopramide may be used to treat tardive dyskinesias induced by other neuroleptic drugs such as prochlorperazine and haloperidol. ${ }^{16}$

Metoclopramide is widely used for treating gastric stasis, nausea and vomiting, and reflux oesophagitis. ${ }^{17}$ It is also being used increasingly for minor symptoms such as nausea, and the dose may be increased above the standard $10 \mathrm{mg}$ three times a day if symptoms are not improved. Metoclopramide has dose dependent kinetics, so an increase in dose will produce a disproportionate rise in plasma concentration owing to the longer half life of the drug. ${ }^{18}$ Moreover, the drug seems to accumulate in patients with renal impairment and also in elderly patients with apparently normal renal function..$^{18}$

Once tardive dyskinesia induced by metoclopramide has developed its management is difficult. Earlier reports suggested that stopping the drug improves symptoms. ${ }^{78}$ Unfortunately, however, symptoms may become worse, and then the temptation may be to try alternative drug treatment. ${ }^{2}$ This should be resisted as further dopamine antagonism, while temporarily lessening symptoms, will only result in their returning in a more serious form. Dopamine depleting agents such as tetrabenazine have a similar effect." The logical choice pharmacologically would be cholinergic drugs (such as physostigmine) or aminobutyric acid inhibitors such as sodium valproate, but results have been disappointing. ${ }^{.1}$ Anticholinergic drugs or levodopa will only worsen symptoms.

Since little can be done once dyskinesia is established the emphasis must be on prevention. Should elderly patients not be given metoclopramide at all? Such a blanket contraindication may not necessarily be justified. An incidence of dyskinesia of one in 1000 patients taking metoclopramide does not necessarily imply a causal relationship. Dyskinesia often occurs spontaneously in elderly patients, just as abnormalities of postural movement were reported in patients with schizophrenia before the use of antipsychotic drugs. ${ }^{19} 20$ Blowers and Borison studied a population of 500 patients in geriatric nursing homes using the abnormal involuntary movement scale for dyskinesias and found spontaneous dyskinesias in $13 \%$-compared with $22 \%$ of patients who had received neuroleptic medication..$^{21}$ No difference was found in the frequency of dyskinesias between men who had received antipsychotic drugs and those who had not. In this study it was the combination of more than one neuroleptic drug that appeared to produce an increased risk of dyskinesias. On the other hand, Jeste and Wyatt's findings indicated that the mean prevalence of dyskinesia among people in long stay institutions was about three times greater in those who had received antipsychotic treatment. ${ }^{19}$ Nevertheless, the Swedish study suggests that metoclopramide is much less likely to be associated with tardive dyskinesia ${ }^{9}$ than antipsychotic therapy. ${ }^{202}$

The conclusion seems plain enough: metoclopramide should not be used for trivial symptoms. Nor should its use be unnecessarily prolonged, because the duration of treatment appears to be at least as important as the total daily dose. For minor symptoms of gastric irritation an antacid is preferable in the short term, though, of course, long term symptomatic treatment is not an alternative to diagnosis of the underlying cause. If no treatable cause is found for nausea an elderly patient should not be denied antiemetic treatment - and it is reasonable to use metoclopramide in the short term provided that the dose is limited to $0.5 \mathrm{mg} / \mathrm{kg}$ a day -10 mg three times a day for most patients.

M L'E ORME

Reader in clinical pharmacology

R C TALlis

University of Liverpool,

New Medical School,

Liverpool L69 3BX

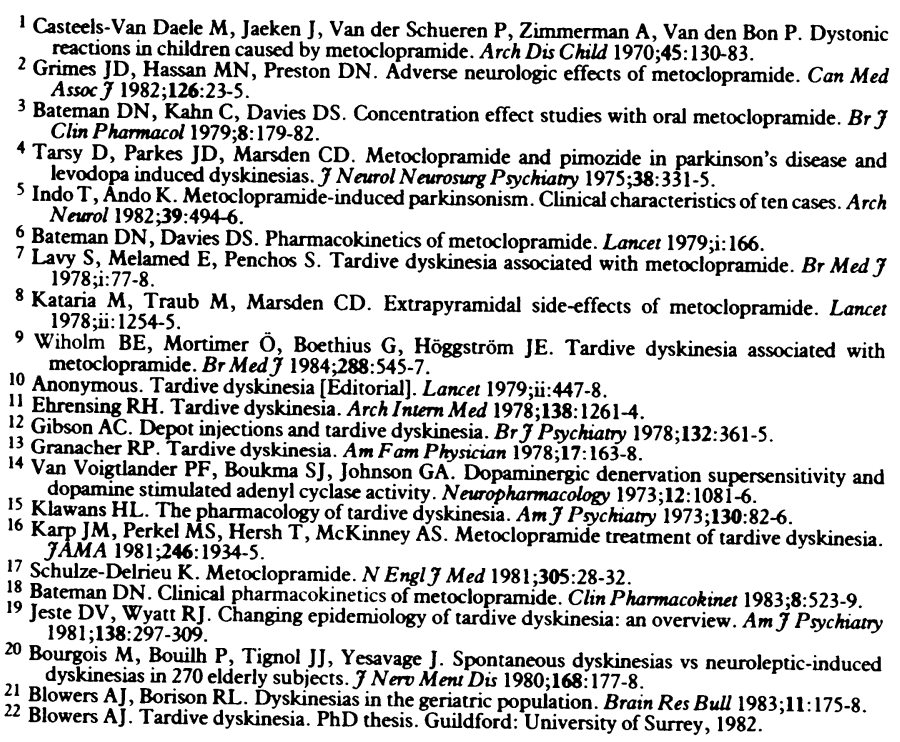

